Edited by:

Misha Tsodyks, Weizmann Institute

of Science, Israel

\section{Reviewed by:}

Israel Nelken, Hebrew University, Israel

John Lisman, Brandeis University, USA

* Correspondence:

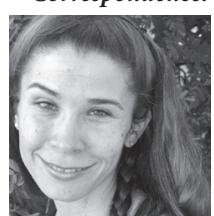

Inés Samengo studied physics in Argentina, and then switched to neuroscience. She spent a year in Berlin, in the group of Andreas Herz, where she also met Ariel Rokem. Together they studied the neural code of insect sensory systems. Back in Argentina, Inés settled as a researcher in Bariloche, where Hugo Eyherabide joined her as a PhD student. Hugo and Inés still interact regularly with Andreas (now in Munich) and Ariel (now in Berkeley), resulting in a quite entertaining intercontinental

collaboration group.

samengo@cab.cnea.gov.ar

\title{
Bursts generate a non-reducible spike-pattern code
}

\author{
Hugo G. Eyherabide ${ }^{1,2}$, Ariel Rokem ${ }^{1,3}$, Andreas V. M. Herz ${ }^{1,4}$ and Inés Samengo ${ }^{1,2 *}$ \\ 1 Bernstein Center for Computational Neuroscience and Institute for Theoretical Biology, Department of Biology, Humboldt \\ Universität, Berlin, Germany \\ 2 Centro Atómico Bariloche and Instituto Balseiro, San Carlos de Bariloche, Argentina \\ ${ }^{3}$ Helen Wills Neuroscience Institute, University of California, Berkeley, USA \\ ${ }^{4}$ Bernstein Center for Computational Neuroscience and Division of Neurobiology, Department of Biology, \\ Ludwig-Maximilians-Universität, Munich, Germany
}

At the single-neuron level, precisely timed spikes can either constitute firing-rate codes or spike-pattern codes that utilize the relative timing between consecutive spikes. There has been little experimental support for the hypothesis that such temporal patterns contribute substantially to information transmission. By using grasshopper auditory receptors as a model system, we show that correlations between spikes can be used to represent behaviorally relevant stimuli. The correlations reflect the inner structure of the spike train: a succession of burst-like patterns. We demonstrate that bursts with different spike counts encode different stimulus features, such that about $20 \%$ of the transmitted information corresponds to discriminating between different features, and the remaining $80 \%$ is used to allocate these features in time. In this spike-pattern code, the what and the when of the stimuli are encoded in the duration of each burst and the time of burst onset, respectively. Given the ubiquity of burst firing, we expect similar findings also for other neural systems.

Keywords: burst spiking, neural code, sensory encoding, information theory, auditory receptor

\section{NEURAL CODES BASED ON SPIKE-TIMING}

Single neurons can encode time-dependent stimuli in several ways. In firing-rate codes the only response feature that carries information is the time-varying firing probability. The information encoded by the temporal correlations between subsequent spikes (if any) is redundant with the one available from the firing rate. The firing probability is a function of the stimulus, often nonlocal and non-linear. Depending on the nature of the transformation between the stimulus and the firing rate, the activity of the neuron may encode the stimulus continuously, or may instead only extract one or a few specific features.

These two extreme situations are depicted in Figures 1A, B. In Figure 1A, the firing probabil- ity is larger than 0 almost everywhere and the cell fires more than one spike in intervals comparable to the duration of the fluctuations in the stimulus. The number of spikes per unit time encodes the stimulus strength through a monotonic transformation. By reading out the number of spikes in an extended time window, typically in the order of tens of milliseconds, downstream neurons have access to a smooth representation of the stimulus.

Figure 1B also exemplifies a firing-rate code, but in this case the firing probability does not represent the entire dynamic range of the stimulus. It rather picks specific features, so that spike generation is only possible shortly after these particular features. In these codes, each single spike suffices 
Firing-rate codes

Neural codes where all the information is encoded in the time-dependent firing rate, additional response properties being irrelevant.

Spike-pattern codes

Neural codes based on patterns of spikes, defined by characteristic correlations in their relative timing.

Inter-spike intervals (ISIs)

The time interval between two subsequent action potentials. to inform downstream neurons of the presence of the relevant feature, and the temporal jitter of individual spikes is often smaller than $1 \mathrm{~ms}$.

The two examples mentioned above fall within the broad class of firing-rate codes. In one extreme, we find codes where the firing rate varies slowly, so the precise timing of individual spikes is not crucial. The neuron encodes the stimulus strength making use of a fairly broad dynamical range, but does so with low temporal precision. In the opposite extreme, the firing rate varies rapidly. As the cell only represents either the presence or absence of the relevant feature, little information is provided about the overall evolution of the stimulus. However, the rapid variation of the time-dependent firing rate provides large amounts of information about the temporal location of the encoded features. These two extreme cases can be framed in a unified mathematical formulation (Rieke et al., 1997). Depending on whether the relevant feature is sharp and brief or flat and broad, the system ranges from a firingrate code based on individual spikes to a firingrate code based on mean spike counts.

Starting with the seminal work of Mainen and Sejnowski (1995), several studies have shown that precise spike timing down to the sub-millisecond regime is important to transmit information about the sensory world. Examples include the insect visual system (Strong et al., 1998), the vertebrate lateral geniculate nucleus (Reinagel and Reid, 2000), the rodent somatosensory thalamus (Montemurro et al., 2007a), and the auditory system, e.g., invertebrate receptor cells (Rokem et al., 2006), vertebrate brainstem neurons (Oertle, 1999) and auditory cortical cells (Heil, 1997).

However, spike-timing based codes can go beyond firing-rate codes. In principle, not only the location of precisely timed individual spikes can transmit information, but also the relative timing between two or more spikes. Such schemes are referred to as relational, or spike-pattern codes. In such codes, the correlations between spikes define patterns, and these patterns are employed to encode stimulus features, each feature corresponding to a particular sequence of inter-spike intervals (ISIs). Reich et al. (2000) and Doiron et al. (2007) showed that different patterns (in this case doublets of spikes separated by ISIs of different durations) were associated with different stimulus features. In these examples, however, different patterns have different instantaneous firing rates, so unless some additional characterization is made, one cannot rule out a purely firing-rate coding scheme.

A neural code that cannot be explained in terms of the instantaneous firing rate requires more complex patterns, including three or more spikes

Siring-rate code
Figure 1 I Schemath encoding
of the stimulus is encoded by the number of spikes fired in an extended time window. The temporal evolution
of the firing probability (shown below) mimics the stimulus. (B) Firing-rate code, where the occurrence of specific
stimulus features (in this case, pronounced upstrokes or downstrokes marked in red) is encoded in the times
at which individual spikes are generated. (C) Spike-pattern code, in which different stimulus features are represented
by different patterns. In this case, a burst-mediated code is shown, where different patterns are distinguished
by their intra-burst spike count. The burst structure presented in this example implies strong temporal correlations
between subsequent spikes. All data were obtained using model neurons.
Source code is available at cabfst28.cnea.gov.ar/ eyherabh/codes.html.


(two or more ISIs). In this paper we review the coding capabilities of a ubiquitous burst-mediated code, where the distinction between different patterns is given by the number of spikes that compose each burst. The set of different codewords consists of bursts containing different numbers of spikes, as exemplified in Figure 1C. In this case, the intra-burst ISI for different codewords remains approximately the same, and the distinction between the different codewords is given by the intra-burst spike count.

Traditionally, burst firing was believed to underlie unconscious regulatory processes during sleep, seizures or anesthesia, and to prevent sensory signals from reaching higher processing stages. This picture emerged from the robust synchronized bursting activity that rises spontaneously in thalamic slices, even in the absence of stimulation (Guillery, 2001). Bursting is also observed in thalamic neurons during sleep, and is disrupted as soon as the subject wakes up, to be replaced by tonic activity during wakefulness. However, in the last decade several studies have shown that burst firing also participates in the representation of the sensory world during the aroused state (Sherman, 2001), as well as in other neural systems (Krahe and Gabbiani, 2004). For example, in the electrosensory lateral line lobe of the weak electric fish, bursts represent lowfrequency events (Oswald et al., 2004), comprising either excitatory or inhibitory stimulus deflections (Metzner et al., 1998). In the mammalian LGN, bursts encode slow stimulus features (Lesica and Stanley, 2004), characterized by high contrast (Reinagel et al., 1999), typical of natural images (Denning and Reinagel, 2005). In the rodent hippocampus, bursting pyramidal place cells represent the location of the animal in the environment, both through the firing rate (Wilson and McNaughton, 2003) and the timing with respect to the theta cycle (O'Keefe and Recce, 1993).

A few studies have specifically explored the information carried by the intra-burst spike count. Two of them involve the mammalian primary visual cortex (DeBusk et al., 1997; Martinez-Conde et al., 2002), where the length of each burst was correlated with the orientation of the stimulus. A theoretical analysis based on a computational model of a cortical pyramidal cell (Kepecs and Lisman, 2003) concluded that the number of spikes inside each burst represented the slope of the incoming stimulus at burst onset. Finally, in tactile sensory neurons in leech (Arganda et al., 2007), the intra-burst spike count represented the velocity of skin displacements. These analyses demonstrate that bursting neurons in different systems represent different stimulus attributes. A common aspect however, is that the information is not only encoded in the time-dependent firing rate, but also in the correlations between spikes.

\section{BURST-MEDIATED CODES IN GRASSHOPPER AUDITORY RECEPTORS}

Grasshoppers communicate with each other by chirping acoustic signals produced by rasping their hind legs across their wings. By analyzing the response of acoustic receptor cells to a broad range of naturalistic and artificial stimuli, we have demonstrated that spiking activity is particularly precise when driven by sound waves whose temporal characteristics coincide with those of the natural songs (Rokem et al., 2006). When these stimuli are played at moderate or loud volume (e.g., nearby sources), receptor neurons have a large probability to elicit bursting responses (Eyherabide et al., 2008). Actually, $93 \%$ of the recorded neurons generated bursts when driven with naturalistic stimuli, whereas none of them bursted in response to signals that varied much faster than the natural songs. Hence, bursting seems to appear in response to behaviorally relevant stimuli only.

If bursts of different spike counts are mapped onto different stimulus features, then the stimuli eliciting shorter bursts must be significantly distinct from the stimuli generating longer bursts. The easiest way to test this hypothesis is to compare the average stimulus preceding single spikes, with that corresponding to doublets, triplets and quadruplets. We defined the burst-triggered average (BTA) corresponding to bursts of $n$ spikes as the average stimulus time course before bursts containing exactly $n$ action potentials (Figure 2A).

In our case, the time-dependent stimulus was the volume (in decibels) of the envelope of a high-frequency input sound wave (Eyherabide et al., 2008). All the BTAs exhibited a pronounced peak on top of a noisy background. Hence, burst production occurred a few milliseconds after a sudden elevation of the sound intensity. The magnitude of the elevation determined the number of spikes in each burst, such that higher stimulus fluctuations elicited longer bursts. In $85 \%$ of all bursting cells, this correspondence was selective: BTAs associated to single spikes, doublets, triplets and quadruplets were significantly different from each other. As a consequence, the number of spikes per burst was a good predictor of the maximal height of the transient intensity fluctuation (Eyherabide et al., 2008). The relationship between stimulus 
A

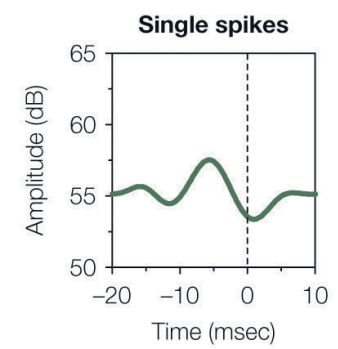

\section{BURST-TRIGGERED AVERAGES}

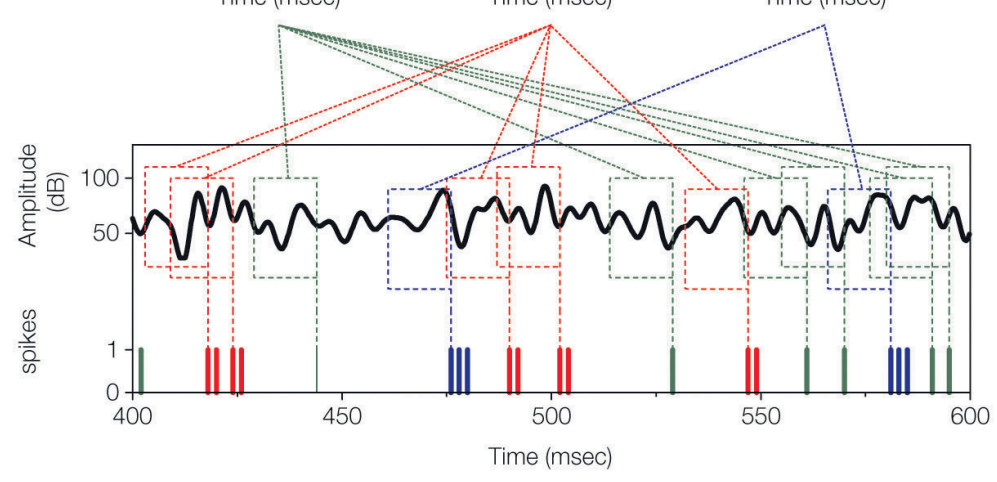

B
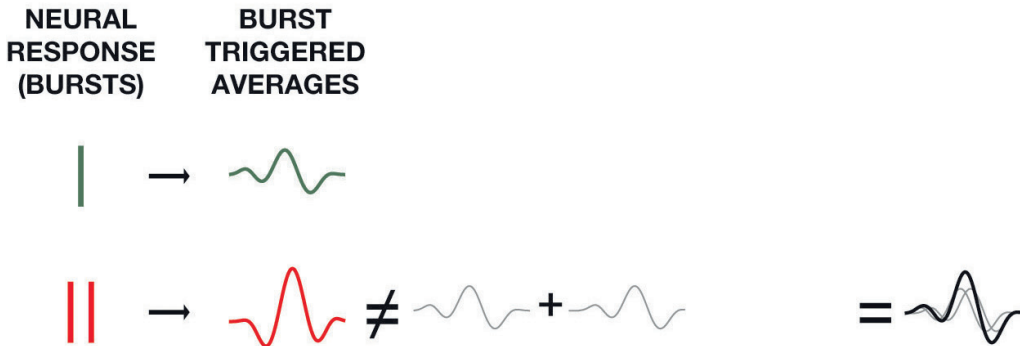

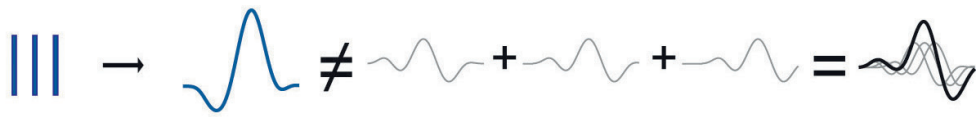

Figure 2 | The burst-triggered averages (BTAs) corresponding to bursts of different durations. (A) Calculation of BTAs. The stimulus stretches preceding bursts of exactly $n$ spikes are aligned with respect to the first spike in the burst, and averaged together. For all cells, BTAs exhibit a central peak, on top of a noisy background. The size of the peak increases with the number of spikesin the burst. (B) The BTA corresponding to doublets is larger than the one that would be obtained by summing up two copies of the BTA of single spikes, displaced from one another by one ISI. The same holds for triplets and quadruplets. intensity and burst duration was not trivial, as sketched in Figure 2B. In 95\% of the bursting neurons, the mean stimulus eliciting a doublet was significantly larger than the stimulus that would be obtained by summing up two copies of the mean stimulus generating isolated spikes, separated by the observed ISI.

Distinguishing between bursts of different durations, hence, allows us to discern between different stimulus features. But how can we be sure that there is no other alphabet that could do a better job? Perhaps there is another set of patterns that also allows one to discern between different stimulus features. We would like to have a criterion to quantify the adequacy of the chosen alphabet, and to rank it with respect to other possible choices. One way would be to measure how much information is lost by only distinguishing between the patterns of the chosen alphabet, and to neglect all additional response features. For a burst code, this amounts to distinguishing between bursts containing different number of spikes, while disregarding the internal temporal structure of each burst beyond its spike count. Not all doublets have exactly the same ISI between their two spikes, and not all triplets have exactly the same ISI sequence. In order to assess the success of the burst alphabet we therefore neglect these differences. Operationally, the spike train is represented as a sequence of symbols that specify the number of spikes in each burst, as sketched in the inset of Figure 3A. All doublets are represented by the same symbol, irrespective of their inner structure. The same holds for triplets, quadruplets, and higher-order bursts. The resulting sequence of symbols can be used to calculate the mutual information between the stimulus and the burst alphabet. If this information is substantially lower than the information in the original spike train, then the inner structure of bursts must be considered as relevant, and the selected alphabet as not appropriate. But this is not the case with our data. In Figure $3 \mathrm{~A}$ we show that the burst representation has almost the same amount of information as the full response. This implies that bursts encode different stimulus features essentially through their intra-burst spike count.

The burst code, though missing several response details, still provides an informative and compact representation of the original spike train. The advantage of such a representation is that now we can explicitly interpret the code. At this stage, we know that the number of spikes in each burst represents the height of the stimulus feature that elicited bursting, whereas the time at which the burst is generated tags the temporal location of this relevant feature. One may therefore wonder how much information corresponds to distinguishing between different features, and how much is accounted for allocating them in time. To show this, we compare the information in the burst train with that of an even more drastically reduced representation of the spike train, in which all bursts are mapped onto the same symbol. No distinctions between 
Mutual information between stimuli and neural responses The amount of knowledge that can be gained about the stimulus by observing the neural activity (and vice versa). This measure can be calculated from the statistical dependence between stimulus and neural response. different types of bursts (and stimulus features) thus remain, as shown in Figure 3B. In the investigated cells this leads to an average reduction of the encoded information of $22 \%$. This percentage represents the 'what' in the stimulus, whereas the complementary fraction accounts for the 'when' (Berry et al., 1997; Borst and Theunissen, 1999; Theunissen and Miller, 1995).

\section{IS THE BURST CODE A FIRING-RATE CODE?}

In our data, the instantaneous firing rate inside different bursts is always approximately the same: we found no significant dependence of the intraburst ISI on the number of spikes per burst. Yet, it could be argued that the burst code is still a firing-rate code, but such that the mean firing rate of the cell should be read out in long intervals. In Rokem et al. (2006) we showed that the larger the time bin used to read out neural responses, the smaller the amount of transmitted information. The loss arises because longer bins have less temporal precision. One could then try using broad time bins, but make them slide along the spike train in very fine steps. Even so, the number of spikes per burst cannot be obtained by counting the spikes inside a window of fixed duration. The code is composed of sequences that alternate between short and long bursts, and the interval between bursts is often comparable to the duration of bursts themselves. Hence, if the window used to count spikes is short, long bursts are not captured. Instead, if a long window is used, then

\section{Transmitted information (bits/sec)}
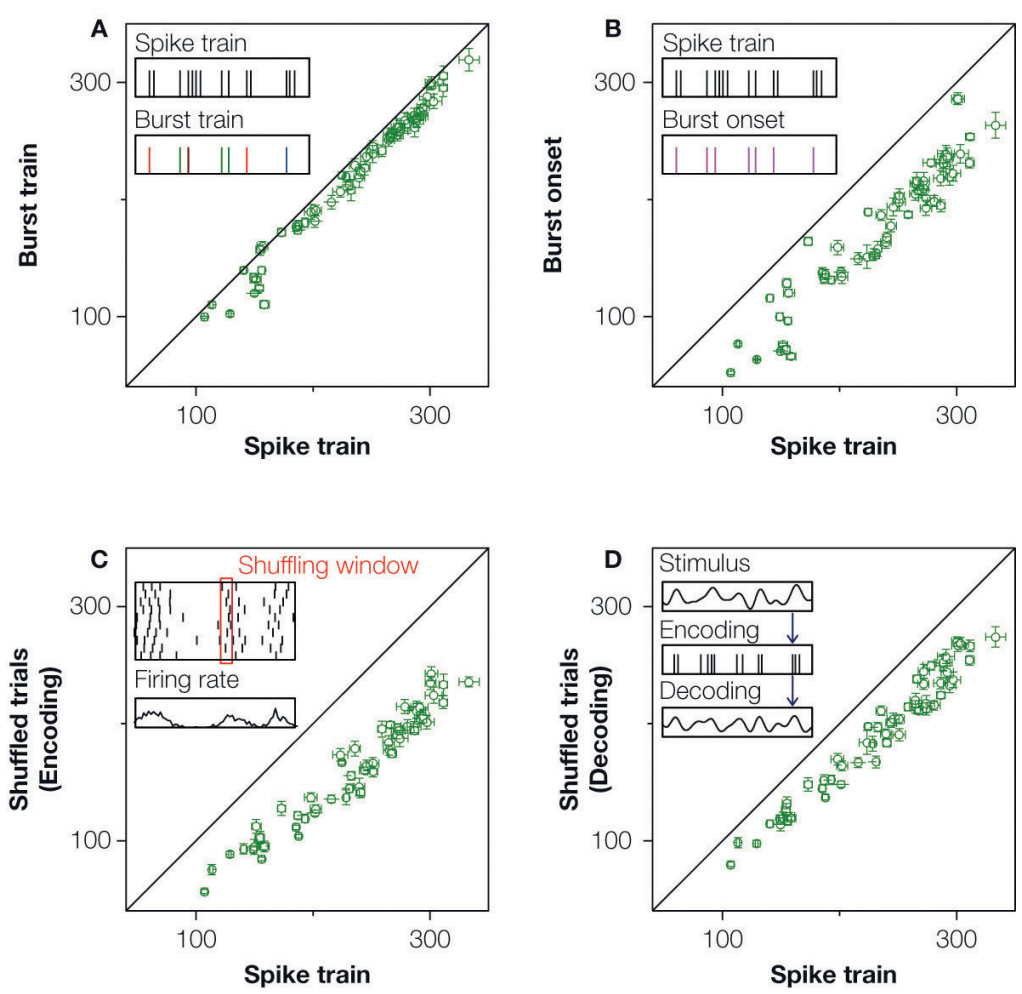

Figure 3 | The information rate carried by manipulated spike trains, for all recorded bursting cells. $x$-axis: original response. y-axis: manipulated response. In all cases, information rates were calculated using the direct method (Strong et al., 1998), with the error estimations from Montemurro et al. (2007b). (A) The spike train is parsed into a sequence of bursts that are only distinguished by their spike count, such that their inner structure is neglected. Inset: green, red, blue and brown symbols in the burst train represent bursts containing 1,2, 3 or 4 action potentials in the original spike train, respectively. The sequence of bursts contains $94 \%$ of the information of the full spike train. (B) If the same symbol is used to represent bursts of different lengths, then distinctions between different stimulus features are no longer possible (see inset). Only temporal information referring to the timing of each feature remains. This leads to a $26 \%$ reduction of the original information ( $22 \%$, if compared to the information transmitted by the burst train). (C) If trials within each fixed time bin are shuffled, all within-trial temporal correlations are destroyed, though the time-dependent firing rate is preserved. As a consequence, the information drops by $32 \%$. (D) If the stimulus is decoded from the spike train (see inset) without taking temporal correlations into account, the information is reduced by $18 \%$. 
Trains of spikes represent information using time with precise calibration.

Scientists reckoned

one millisecond

affected a representation.

Is it each single spike that sustains, with its timing, a code in the brain? Or is the relative time

between spikes that defines

a pattern-based reference frame?

Correlational codes have been cursed as the ones whose transparency is worst. But here we show

that as far as we know

grasshoppers use patterns of bursts.

For a spike means a different thing

than a doublet of spikes, or a string.

So a burst, with its length,

codes for stimulus strength

with a non-linear map in between.

And the bursts in our brain are employed

to build patterns minutely deployed.

If we shuffle them out,

we show here, no doubt,

the information they carry is destroyed.

Just as words when exquisitely chained

make a poem a lyrical game,

just as notes, when combined,

build up music, sublime,

so do bursts become thoughts in the brain.

Before closing, allow us to first

tell you how to manage your thirst:

If you want to think clear

after drinking a beer

make sure to let out a burst.

Figure 4 | A burst of rhymes to close,

and complement the prose.

several short bursts are mistaken as a single longer burst. The same problem arises if one tries to convolve the spike train with a smooth bell-shaped weight function, as for example, a Gaussian kernel of fixed width. Therefore, bursts do not constitute a convolved firing-rate code either.

More importantly, the burst code found in grasshopper receptors uses not only the precision of individual spikes, but also the correlations between spikes. Two subsequent spikes may or may not be part of the same burst, depending on the size of the ISI separating them. Hence, it is their relative timing that matters. In firing-rate codes, however, correlations between spikes can be entirely explained in terms of the time-varying firing probability. Therefore, correlations make no additional contribution to the encoding or decoding of information, beyond the information available in the firing probability.
The impact of correlations on the encoding of sensory information can be assessed by shuffling trials in the neural response, for each fixed time bin, as highlighted in the inset of Figure 3C. By doing so, the time-dependent firing rate is preserved, while the within-trial correlations are abolished. If the shuffled spike train contains significantly less information than the real spike train, then removing the correlations has a negative impact in the transmitted information. This approach was proposed as a measure of conditional independence in the framework of population coding (Schneidman et al., 2003). When later employed to assess the role of temporal correlations in single neurons in the rat thalamus (Montemurro et al., 2007a), spike patterns played a minor role. They only increased the total information by $6 \%$ and the remaining $94 \%$ was entirely attributable to the time-dependent firing rate. Thus, most information was transmitted by precisely timed single spikes. In grasshopper receptor cells, however, spike patterns play a more important role, as shown in Figure 3C. The shuffled responses carried 32\% less information than the full spike train. The constraints imposed by correlations, hence, resulted in a substantially improved coding scheme as compared to the one that would be obtained by independent time bins.

A complementary approach is to assess the effect of correlations in neuronal decoding (Latham and Nirenberg, 2005). This formulation allows us to evaluate whether the original stimulus can be decoded from the neural responses equally well, if within-trial temporal correlations are neglected. Since we do not know which decoding scheme is employed by downstream neurons in the brain, we use optimal Bayesian decoding, guaranteed to perform at least as well as any biological decoding. Figure 3D shows that neglecting the correlations has a detrimental effect in the decoded information of almost 20\%, an amount that is very similar to the information needed to discriminate between different stimulus features, see Figure 3B. Therefore, grasshopper auditory responses can only be decoded properly if their correlational structure is taken into account as summarized in Figure 4.

\section{CONCLUSIONS}

One of the central problems in neuroscience is to understand the way in which sensory information is represented in the nervous system. The objective is to know the general principles of the encoding scheme, and to have an explicit dictionary connecting stimulus features with response characteristics. This study addresses both issues. By using information-theoretical quantities, we were 
able to extract some general principles governing the representation of acoustic information in grasshopper receptors. We demonstrated that the code is based on spike patterns, and that the information conveyed by these patterns cannot be accounted for by a rapidly varying firing rate. The code is structured in bursts, and the distinctive feature discerning between different types of bursts is the number of spikes they contain. The time of burst onset represents the temporal location of a certain stimulus feature, and the intra-burst spike count discriminates between different types of features. Using theoretical techniques, we quantified the fraction of information that corresponds to these two aspects individually. By thoroughly examining the correspondence between bursts and stimuli, we revealed the meaning of each pattern in terms of the height of sudden amplitude fluctuations. The non-linear transformation between spike count and stimulus amplitude provides additional evidence that spike patterns must be read out as compound codewords, and not as a collection of single spikes.

Our model system, hence, provides an example of a correlation-based neural code whose building blocks are bursts of spikes. Burst firing is ubiquitous in the nervous systems of both vertebrates and invertebrates. Our study suggests that the role of bursts in sensory representations might be even more relevant than previously thought.

\section{ACKNOWLEDGMENTS}

This work was supported by the Alexander von Humboldt Foundation, the Deutsche Forschungsgemeinschaft (SFB 618), the Consejo de Investigaciones Científicas y Técnicas, the German Federal Ministry of Education and Research (BCCN Berlin and BCCN Munich), the Israeli Ministry of Science, the Minerva Foundation of the Max Planck Society, and the Agencia de Promoción Científicas y Tecnológica of Argentina.

\section{REFERENCES}

Arganda, S., Guantes, R., and de Polavieja, G. G. (2007). Sodium pumps adapt spike bursting to stimulus statistics. Nat. Neurosci. 10, 1467-1473.

Berry, M. J., Warland, D. K., and Meister, M. (1997). The structure and precision of retinal spike trains. Proc. Natl. Acad. Sci. USA 94, 5411-5416.

Borst, A., and Theunissen, F. E. (1999). Information theory and neural coding. Nat. Neurosci. 2, 947957.

Denning, K. S., and Reinagel, P. (2005). Visual Control of Burst Priming in the Anesthetized Lateral Geniculate Nucleus. J. Neurosci. 25, 35313538.

DeBusk, B. C., DeBruyn, E. J., Snider, R. K., Kabara, J. F., Bonds, A. B. (1997). Stimulus-Dependent Modulation of Spike Burst Length in Cat Striate Cortical Cells. J. Neurophysiol. 78, 199-213.

Doiron, B., Oswald, A. M. M., and Maler, L. (2007). Interval Coding. II. Dendrite-Dependent Mechanisms. J. Neurophysiol. 97, 2744-2757.

Eyherabide, H. G., Rokem, A., Herz, A. V. M, and Samengo, I. (2008). Burst firing is a neural code in an insect auditory system. Front. Comput. Neurosci. 2:3.

Guillery, W. (2001). Exploring the thalamus. New York, Academic Press.

Heil, P. (1997). Auditory cortical onset responses revisited. I. First-spike timing. J. Neurophysiol. 77, 2616-2641.

Kepecs, A., and Lisman, J. (2003). Information encoding and computation with spikes and bursts. Network 14, 103-118.
Krahe, R., and Gabbiani, F. (2004). Burst firing in sensory systems. Nat. Rev. Neurosci. 5, 13-23.

Latham, P. E., and Nirenberg, S. (2005). Synergy, redundancy and independence in population codes, revisited. $J$. Neurosci. 25, 5195-5206.

Lesica, N. A., and Stanley, G. B. (2004). Encoding of natural scene movies by tonic and burst spikes in the lateral geniculate nucleus. J. Neurosci. 24, 10731-10740.

Mainen, Z. F., and Sejnowski, T. J. (1995). Reliability of spike timing in cortical neurons. Science 268, 1503-1506.

Martinez-Conde, S., Macknik, S. L., and Hubel, D. H. (2002). The function of bursts of spikes during visual fixation in the awake primate lateral geniculate nucleus and primary visual cortex. Proc. Natl. Acad. Sci. U.S.A. 99, 13920-13925.

Metzner, W., Koch, C., Wessel, R., and Gabbiani, F. (1998). Feature extraction by burst-like spike patterns in multiple sensory maps. J. Neurosci. $18,2283-2300$.

Montemurro, M. A., Panzeri, S., Maravall, M., Alenda, A., Bale, M. R., Bramilla, M., and Petersen, R. S. (2007a). Role of precise spike timing in coding of dynamic vibrissa stimuli in somatosensory thalamus. J. Neurophysiol. 98, 1871-1882.

Montemurro, M. A., Senatore, R., and Panzeri, S. (2007b). Tight data-robust bounds to mutual information combining shuffling and model selection techniques. Neural Comput. 19, 2913-2957.
Oertle, D. (1999). The role of timing in the brain stem of vertebrate auditory nuclei. Annu. Rev. Physiol. 61, 497-519.

O'Keefe, J., and Recce, M. L. (1993). Phase relationship between hippocampal place units and the EEG theta rhythm. Hippocampus 3, 317-330.

Oswald, A. M. M., Chacron, M. J., Doiron, B., Bastian, J., and Maler, L. (2004). Parallel processing of sensory input by bursts and isolated spikes. $J$. Neurosci. 24, 4351-4362.

Reich, D. S., Mechler, F., Purpura, K. P., and Victor, J. D. (2000). Interspike intervals, receptive fields, and information encoding in primary visual cortex. $J$. Neurosci. 20, 1964-1974.

Reinagel, P., Godwin, D., Sherman, S. M. and Koch, C. (1999). Encoding of visual information by LGN bursts. $J$. Neurophysiol. 81, 2558-2569.

Reinagel,P., and Reid,R.C.(2000). Temporal coding of visual information in the thalamus. J. Neurosci. 20, 5392-5400.

Rieke, F., Warland, D., de Ruyter van Steveninck, R., and Bialek, W. (1997). Spikes: Exploring the Neural Code. Cambridge, MIT Press.

Rokem, A., Watzl, S., Gollisch, T. Stemmler, M., Herz, A. V. M., and Samengo, I. (2006). Spike-timing precision underlies the coding efficiency of auditory receptor neurons. J. Neurophysiol. 95, 2541-2552.

Schneidman, E., Bialek, W., and Berry, M. J. (2003). Synergy, redundancy, and independence in population codes. $J$. Neurosci. 23, 11539-11553.
Sherman, S. M. (2001). Tonic and burst firing: dual modes of thalamocortical relay. Trends Neurosci. 24, 122-126.

Strong, S. P., Koberle, R., de Ruyter van Steveninck, R. R., and Bialek, W. (1998). Entropy and information in neural spike trains. Phys. Rev. Lett. 80, 197-200.

Theunissen, F., and Miller, J. P. (1995). Temporal encoding in nervous systems: a rigorous definition. J. Comput. Neurosci. 2, 149-162.

Wilson, M. A., and McNaughton, B. L. (2003). Dynamics of the hippocampal ensemble code for space. Science 261, 1055-1058.

Conflict of Interest Statement: The authors declare that the research was conducted in the absence of any commercial or financial relationships that could be construed as a potential conflict of interest.

Received: 01 February 2009; paperpending published: 14 February 2009; accepted: 21 February 2009; published: 01 May 2009. Citation: Front. Neurosci. (2009) 3,1: 8-14. doi: 10.3389/neuro.01.002.2009

Copyright (C) 2009 Eyherabide, Rokem, Herz and Samengo. This is an openaccess article subject to an exclusive license agreement between the authors and the Frontiers Research Foundation, which permits unrestricted use, distribution, and reproduction in any medium, provided the original authors and source are credited. 